\title{
Renal Association Clinical Practice Guideline in Mineral and Bone Disorders in CKD
}

\author{
Dr Simon Steddon ${ }^{\mathrm{a}}$ and Dr Edward Sharples ${ }^{\mathrm{b}}$ \\ aConsultant Nephrologist, Guy's and St Thomas' NHS Foundation Trust, London \\ ${ }^{b}$ Consultant Nephrologist, Churchill Hospital, Oxford
}

\section{Key Words}

mineral bone disorders - chronic kidney disease · parathyroid hormone $\cdot$ calcium $\cdot$ phosphate $\cdot$ phosphate binder

\section{Introduction}

These guidelines are an updated version of the previous 'Complications of CKD Guidelines - Renal Bone Disease'. For this updated version, a systematic literature review was performed using www.pubmed.gov, focusing on the topics of:

Mineral bone disorder

Renal osteodystrophy

Hyperphosphat(a)emia

Calcium

PTH

Vascular calcification

Phosphate binder

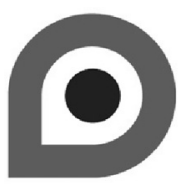

NHS Evidence Accreditation Mark
The search covered the period from January 2006 to November 2009. Articles not written in English were not assessed. Articles available in abstract forms; letters; case reports; editorials or review articles were also excluded. Articles were assessed for relevance to the guideline topic, eligibility for inclusion in the evidence base for that guideline and methodological quality. Articles were considered of particular relevance if they were describing:

- Prospective randomised or quasi-randomised trials;

- Controlled trials;

- Meta-analysis of several trials;

- Cochrane systematic reviews.

As with other Renal Association Clinical Practice Guidelines, the modified GRADE (Grades of recommendation, assessment, development and evaluation) system is used throughout. This system defines both the strength of the recommendations and the level of evidence upon which each is based [1]. It classifies recommendations as 'strong' (Grade 1) or 'weak' (Grade 2) based upon benefits, risks, burden and cost. The quality or level of

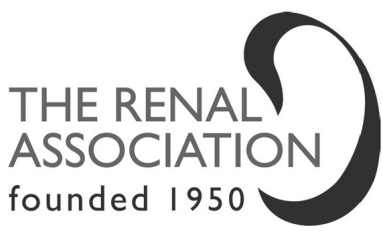

Dr Simon Steddon and Dr Edward Sharples

Email: Dr Simon Steddon at Simon.Steddon@gstt.nhs.uk or Dr Edward Sharples at Edward.Sharples@orh.nhs.uk
(C) $2011 \mathrm{~S}$. Karger AG, Basel

1160-2110/11/1185-000145\$38.00/0

Accessible online at: www.karger.com/nec 
evidence is designated as high (Grade A), moderate (Grade B), low (Grade C) or very low (D) depending on factors such as study design, directness of evidence and consistency of results.

Recommendations take into account the KDOQI Guidelines for management of bone disease in CKD [2] as well as the guidelines for identification and management of CKD issued by NICE [3].

The recent publication of the KDIGO (Kidney Disease: Improving Global Outcomes) evidence based guidelines for the prevention, diagnosis, evaluation, and treatment of CKD: MBD [4], has led to a re-evaluation of the current evidence and targets used in the management of MBD. KDIGO agreed to include only randomised controlled trials of at least 6 months duration, with sample size of at least 50 patients in systematic reviews. A response to the KDIGO guidelines has been published on behalf of the Renal Association, and the response to these guidelines has had a significant impact on the development of these new guidelines. It is important to note that KDIGO focused their guideline parameters on the minimisation of risk based on best current evidence, rather than targeting ideal clinical practice targets for biochemical parameters.

\section{References}

1 Atkins D, Best D, Briss P, et al. GRADE Working Group. Grading quality of evidence and strength of recommendations. BMJ 2004;328:1490

2 National Kidney Foundation. KDOQI Clinical Practice Guidelines for Bone Metabolism and Disease in Chronic Kidney Disease. Am J Kidney Dis 2003;42(Suppl 3):S1-202

3 National Collaborating Centre for Chronic Conditions. Chronic kidney disease: national clinical guideline for early identification and

management in adults in primary and secondary care. London: Royal College of Physicians, Sept 2008

4 KDIGO Clinical Practice Guideline for the Diagnosis, Evaluation, Prevention, and Treatment of Chronic Kidney Disease - Mineral and Bone Disorder. Kidney Int 2009;76(Suppl 113):S1-132 


\section{Summary of Clinical Practice Guidelines on CKD-MBD}

\section{CKD-Mineral and Bone Disorders (Guideline CKD-MBD 1.1)}

\section{Guideline 1.1 CKD-MBD: Monitoring of biochemical parameters}

We suggest that serum levels of calcium, phosphate, alkaline phosphatase, PTH and calcidiol $(25(\mathrm{OH}) \mathrm{D})$ should be monitored in patients with CKD stage 3-5, and patients on dialysis, with a frequency based on stage, rate of progression and whether specific therapies have been initiated. In general, it is recommended that therapeutic decisions are based on trends, rather than a single laboratory value, and that they take into account the entire available dataset, rather than isolated variables (not graded).

\section{CKD-Mineral and Bone Disorders (Guidelines CKD-MBD 2.1-2.2)}

Guideline 2.1 CKD-MBD: Serum calcium in patients with CKD stage 3-5 (not on dialysis)

We suggest that serum calcium, adjusted for albumin concentration, in patients with CKD stage 3-5 should be kept within the normal reference range for the laboratory used. (2D)

Guideline 2.2 CKD-MBD: Serum calcium in dialysis patients (stage 5D)

We suggest that serum calcium, adjusted for albumin concentration, should be maintained within the normal reference range for the laboratory used, measured before a 'short-gap' dialysis session in haemodialysis patients. Ideally, adjusted serum calcium should be maintained between 2.2 and $2.5 \mathrm{mmol} / \mathrm{L}$, with avoidance of hypercalcaemic episodes. (2D)

\section{CKD-Mineral and Bone Disorders (Guidelines CKD-MBD 3.1-3.2)}

Guideline 3.1 CKD-MBD: Serum phosphate in patients with CKD 3-5 (not on dialysis)

We suggest that serum phosphate in patients with CKD stage $3 \mathrm{~b}-5$ should be maintained between 0.9 and $1.5 \mathrm{mmol} / \mathrm{L} .(2 \mathrm{C})$
Guideline 3.2 CKD-MBD: Serum phosphate in dialysis patients (stage $5 \mathrm{D}$ )

We suggest that serum phosphate in dialysis patients, measured before a 'short-gap' dialysis session in haemodialysis patients, should be maintained between 1.1 and $1.7 \mathrm{mmol} / \mathrm{L}$. (2C)

\section{CKD-Mineral and Bone Disorders (Guidelines CKD-MBD 4.1-4.2)}

\section{Guideline 4.1 CKD-MBD: Serum PTH in patients} with CKD $3 b-5$ (not on dialysis)

We suggest that treatment is considered in patients with CKD stages $3 \mathrm{~b}-5$ not on dialysis therapy in whom serum PTH levels are progressively increasing and remain persistently higher than the upper reference limit for the assay, despite correction of modifiable factors. (2C)

Guideline 4.2 CKD-MBD: Serum PTH in patients on dialysis (stage 5D)

\section{Guideline 4.2.1 CKD-MBD: Target range of serum} $P T H$ in patients on dialysis

We suggest that the target range for parathyroid hormone measured using an intact PTH assay should be between 2 and 9 times the upper limit of normal for the assay used. (2C)

Guideline 4.2.2 CKD-MBD: Trends in serum PTH in patients on dialysis

We suggest that marked changes in PTH levels in either direction within this range should prompt an initiation or change in therapy to avoid progression to levels outside this range. (2C)

\section{Summary of Audit Measures in CKD-MBD}

1. Serum calcium in dialysis patients (pre-dialysis for haemodialysis patients).

2. Serum phosphate in dialysis patients (pre-dialysis for haemodialysis patients).

3. Proportion of PTH values within range $0 / 4,1 / 4$, $2 / 4,3 / 4$, and $4 / 4$ of the 4 annual measurements of PTH in CKD stage 5D patients.

4. Percentage of patients with all parameters (calcium/ phosphate/PTH) within target range. 
Rationale for Clinical Practice Guidelines in CKD-MBD

\section{CKD-Mineral and Bone Disorders (Guideline CKD-MBD 1.1)}

\section{Guideline 1.1 CKD-MBD: Monitoring of biochemical parameters}

We suggest that serum levels of calcium, phosphate, alkaline phosphatase, PTH and calcidiol $(25(\mathrm{OH}) \mathrm{D})$ in patients with CKD stage $4-5$, should be monitored, with a frequency based on stage, rate of progression and whether specific therapies have been initiated. In general, it is recommended that therapeutic decisions are based on trends, rather than a single laboratory value, and that they take into account the entire available data set, rather than isolated variables (ungraded).

\section{Rationale}

Abnormal biochemical parameters of CKD-MBD can be observed relatively early in the progression of chronic kidney disease, although the rate of change and severity of abnormalities are highly variable among patients. Evidence from large observation studies, including the STEEKD (Study to Evaluate Early Kidney Disease) which included 1800 patients with CKD stages 3-5, demonstrate that although PTH may be elevated at eGFR $>80 \mathrm{~mL} / \mathrm{min}$, the prevalence of hyperphosphataemia is relatively rare until eGFR falls below $30 \mathrm{~mL} / \mathrm{min}$ [1]. A similar prevalence of hypocalcaemia was observed.

There is no data showing that routine measurement of bone parameters improves patient outcomes. However, it is reasonable to suggest that these parameters are measured with frequency of monitoring based on stage, rate of progression and whether specific therapies have been initiated. A suggested frequency of monitoring is suggested in table 1, but will depend on initial values, trends of change and adjustments to treatment. Alkaline phosphatase is a useful adjunct test and may increase the predictive power of biochemical monitoring when taken into consideration concomitantly with PTH.
Despite epidemiological data that links increased calcium-phosphate product with reduced patient survival, the value of this product is driven predominately by phosphate concentration. In line with the KDIGO guideline, we recommend that calcium-phosphate product is not routinely used to guide treatment of CKD-MBD and no longer included in audit data collection.

Although the prevalence of mild hyperparathyroidism in people with a reduced GFR is higher than normal kidney function, the significance of such modest elevations in PTH concentration remains unknown [2]. There are also cost and logistical implications for the routine measurement of serum $\mathrm{PTH}$ in all CKD stage 3. This is reflected in NICE guidance not to measure unless there is another clinical indication such as hypercalcaemia or symptoms suggestive of hyperparathyroidism [3]. However, such problems often occur late or are difficult to identify. A pragmatic approach is to measure serum PTH at baseline in all those with progressive CKD stage $3 b$ or higher, as values in need of therapeutic attention are more likely to be identified.

Justification for the identification and treatment of vitamin D 'substrate' deficiency assumes independent benefits for non-activated vitamin D (ergocalciferol, colecalciferol) through tissue conversion of 25-hydroxyvitamin D (calcidiol) to 1,25-dihydroxyvitamin D (calcitriol). Vitamin D insufficiency appears common in the CKD population, perhaps the consequence of co-morbidity limiting access to sunlight, and may be a cause of early increases in PTH concentration [3]. In addition, numerous observational studies show an association between low vitamin D levels and adverse clinical outcomes [5]. Although the benefits are unconfirmed, a reasonable case exists for the measurement of vitamin $\mathrm{D}$ in $\mathrm{CKD}$. A pragmatic approach is to measure 25-hydroxyvitamin $\mathrm{D}$ at baseline in CKD stage $3 \mathrm{~b}$ and above, with a view to correction of insufficiency or deficiency $(>75 \mathrm{nmol} / \mathrm{L}=$ repletion, $37.5-75 \mathrm{nmol} /$ $\mathrm{L}=$ insufficiency, $<37.5 \mathrm{nmol} / \mathrm{L}=$ deficiency). Vitamin $\mathrm{D}$ levels need to be interpreted in the context of the

Table 1. Suggested frequency of biochemical testing in CKD-MBD.

\begin{tabular}{llllll}
\hline CKD stage & Calcium & Phosphorus & PTH & Alkaline phosphatase & Calcidiol (25(OH)D) \\
\hline 3b Progressive & Every 6-12 months & Every 6-12 months & Baseline & Every 6-12 months & Baseline \\
4 & Every 3-6 months & Every 3-6 months & Every 6-12 months & Every 3-6 months & Baseline $^{*}$ \\
5 & Every 1-3 months & Every 1-3 months & Every 3-6 months & Every 1-3 months & Baseline $^{*}$ \\
5D & Every 1-3 months & Every 1-3 months & Every 3-6 months & Every 1-3 months & Baseline $^{*}$ \\
\hline
\end{tabular}

${ }^{\star}$ If not receiving an active vitamin D sterol such as calcitriol, alfacalcidol, or paricalcitol. 
patient's overall clinical condition, other biochemical abnormalities, and the pre-existing therapy. Repeat testing will be determined by baseline value and therapeutic interventions. There is insufficient evidence to suggest measurement or repletion in patients who are already

\section{References}

1 Levin A, Bakris G, Molitch M, et al. Prevalence of abnormal serum vitamin $\mathrm{D}, \mathrm{PTH}$, calcium and phosphorus in patients with chronic kidney disease: results of a study to evaluate early kidney disease. Kidney Int 2007;71:31-38

2 Vassalotti J, Uribarri J, Chen S, et al. Trends in mineral metabolism: Kidney Early Evaluation Program (KEEP) and the National Health and Nutritional Examination Survey (NHANES) 1999-2004. Am J Kidney Dis 2008; $51:$ S56-58

3 National Collaborating Centre for Chronic Conditions. Chronic kidney disease: national clinical guideline for early identification and management receiving an active vitamin D sterol such as calcitriol, alfacalcidol, or paricalcitol. It must be pointed out, however, that repletion strategies are currently ill-defined and may actually have very little effect on vitamin D stores. in adults in primary and secondary care. London: Royal College of Physicians, Sept 2008

4 Craver L, Marco MP, Martínez I, et al. Mineral metabolism parameters throughout chronic kidney disease stages 1-5 - achievement of K/DOQI target ranges. Nephrol Dial Transplant 2007;22:1171-1176

5 Al-Badr W, Martin K. Vitamin D and Kidney Disease. Clin J Am Soc Nephrol 2008;3(5):1555-1560 


\section{CKD-Mineral and Bone Disorders (Guidelines CKD-MBD 2.1-2.2)}

\section{Guideline 2.1 CKD-MBD: Serum calcium in patients} with CKD stage 3-5 (not on dialysis)

We suggest that serum calcium, adjusted for albumin concentration, in patients with CKD stage $3-5$ should be kept within the normal reference range for the laboratory used. (2D)

Guideline 2.2 CKD-MBD: Serum calcium in dialysis patients (stage 5D)

We suggest that serum calcium, adjusted for albumin concentration, should be maintained with the normal reference range for the laboratory used, measured before a 'short-gap' dialysis session in haemodialysis patients. Ideally, adjusted serum calcium should be maintained between 2.2 and $2.5 \mathrm{mmol} / \mathrm{L}$, with avoidance of hypercalcaemic episodes. (2D)

\section{Rationale ( for 2.1-2.2)}

The measurement of serum calcium, adjusted for albumin concentration, is susceptible to all the problems of inter-assay variation, and in addition, there are several different formulae for correction for albumin concentration. Calcium should be controlled to avoid symptomatic hypocalcaemia and hypocalcaemia driven stimulation of the parathyroid glands. There is some evidence that, in addition to known associations between hyperphosphataemia and mortality, calcium concentrations have an independent association with relative mortality risk [1]. In a large retrospective review of over 40,000 haemodialysis patients, all cause mortality was relatively higher the higher the corrected calcium level, after adjustment for gender, age, dialysis vintage and diabetes [2].

\section{References}

1 Kovesdy C, Kuckmak O, Lu JL, Kalantar-Zadeh K. Outcomes associated with serum calcium level in men with non-dialysis-dependent chronic kidney disease. Clin J Am Soc Nephrol 2010;5:468-476

2 Wald R, Sarnak M, Tighiouart H, et al. Disordered mineral metabolism in haemodialysis patients: An analysis of the cumulative effects in the Haemodialysis Study (HEMO). Am J Kidney Dis 2008;52:531-540

3 Tentori F, Blayney M, Albert JM, et al. Mortality risk for dialysis patients with different levels of serum calcium, phosphorus, and PTH: The Dialysis Outcomes and Practice Patterns Study (DOPPS). Am J Kidney Dis 2008;52:519-530
Observational data from the DOPPS study [3] show a similar relationship between calcium and mortality. In 17,236 haemodialysis patients from 307 participating centres in the US, Europe and Japan, all-cause mortality was associated with a increased RR for each $0.25 \mathrm{mmol} / \mathrm{L}$ increase in calcium. There was an increased relative risk associated with calcium levels less than $2.2 \mathrm{mmol} / \mathrm{L}$. With this observational data, and in the absence of randomised controlled trials demonstrating reduced mortality associated with corrected calcium at the lower end of the normal range, we do not recommend the serum calcium should be below $2.2 \mathrm{mmol} / \mathrm{L}$. Cumulative time dependent analysis of the DOPPS study shows that calcium values $>2.65$ are associated with significant hazard ratio for all cause mortality (1.66 (1.09-2.55)).

There is evidence that calcium load and episodes of hypercalcaemia are associated with a relative increase in vascular calcification and arterial stiffness, although, at present, randomised controlled trials of non-calcium binders versus calcium binders have not demonstrated a significant reduction in mortality, despite a reduction in the amount, and progression, of vascular calcification. It would be recommended to review patients requiring high doses of calcium-based binders and consider switching to avoid excess calcium loading and risk of hypercalcaemia.

It is unclear whether the introduction of the calcimimetic agent cinacalcet, which NICE recommend reserving for patients who are unsuitable for surgical parathyroidectomy [4], should alter the target range for calcium. Cinacalcet improves calcium and phosphate levels as well as controlling PTH, with long term studies showing persistent improvements in hyperphosphataemia and a low incidence of hypercalcaemia [5].
4 National Collaborating Centre for Chronic Conditions. Chronic kidney disease: national clinical guideline for early identification and management in adults in primary and secondary care. London: Royal College of Physicians, Sept 2008

5 Sprague S, Evenepoel P, Curzi M, et al. Simultaneous control of PTH and CaxP is sustained over three years of treatment with cinacalcet $\mathrm{HCl}$. Clin J Am Soc Nephrol 2009;4:1465-1476 


\section{CKD-Mineral and Bone Disorders (Guidelines CKD-MBD 3.1-3.2)}

\section{Guideline 3.1 CKD-MBD: Serum phosphate in patients with CKD 3-5 (not on dialysis)}

We suggest that serum phosphate in CKD patients stage $3 \mathrm{~b}-5$ should be maintained between 0.9 and $1.5 \mathrm{mmol} / \mathrm{L}$. (2C)

\section{Guideline 3.2 CKD-MBD: Serum phosphate in} dialysis patients (stage 5D)

We suggest that serum phosphate in dialysis patients, measured before a 'short-gap' dialysis session in haemodialysis patients, should be maintained between 1.1 and $1.7 \mathrm{mmol} / \mathrm{L}$. (2C)

\section{Rationale (for 3.1-3.2)}

Hyperphosphataemia is one of the commonest biochemical abnormalities through the course of CKD and poses significant challenges for management. Epidemiological and observational studies have shown a strong association between hyperphosphataemia and mortality in dialysis patients. Indeed, it is now recognised, from the Framingham Offspring study, that serum phosphate is associated with the risk of cardiovascular disease in the general population [1].

Elevated phosphate levels in CKD contribute to the development of low calcitriol levels, hyperparathyroidism, abnormal bone remodelling, and vascular calcification.

Hyperphosphataemia is associated with increased morbidity and mortality in dialysis patients. In a large cross-sectional and retrospective analysis of over 40,000 haemodialysis patients, the lowest relative risk of death was associated with serum phosphate concentrations between $0.97-1.6 \mathrm{mmol} / \mathrm{L}$ [2]. Renal Registry data shows a similar relationship, with the lowest risk of death at serum phosphate concentrations between 1.1 and $1.8 \mathrm{mmol} / \mathrm{L}$. A recent systematic review of the evidence of an association between hyperphosphataemia and mortality confirmed that, despite wide heterogeneity between studies, there was a clear association between hyperphosphataemia and mortality in both haemodialysis and peritoneal dialysis patients [3]. The cut-off value for this association was between $1.5-2.4 \mathrm{mmol} / \mathrm{L}$, but individual studies used different reference ranges, limiting potential for accurate meta-analysis. This relationship was also observed in pre-dialysis CKD patients. The 2009 Renal Registry report shows that for haemodialysis patients median phosphate is 1.5 , and there has been a progressive lowering of median concentrations over time. In view of this, and the strong epidemiological evidence, we recommend lowering the upper limit for phosphate to $1.7 \mathrm{mmol} / \mathrm{l}$.

There is no specific evidence that lowering serum phosphate to a particular level leads to an improved clinical outcome in patients with CKD, so recommended treatment goals are based on evidence from observational data. However, a retrospective US study of 13,792 patients on haemodialysis demonstrated a reduced relative risk of mortality in patients who achieved serum phosphate concentrations within the K/DOQI guideline range, when compared to patients who did not [4]. The use of a phosphate-restricted diet in combination with oral phosphate binders has become well established in the management of CKD 4-5D.

There is insufficient data from randomised controlled trials that any specific oral phosphate binder impacts on individual patient outcome, and hence the choice of oral binders should be individualised, based on the effects of available agents on a range of clinical parameters, especially the avoidance of hypercalcaemia, rather than solely focused on serum phosphate alone. The Dialysis Clinical Outcomes Revisited (DCOR) study randomised 2103 patients to either sevelamer or a calcium-based phosphate binder, with a mean follow-up of 20 months [5]. There was no difference in all-cause or cardiovascular mortality between the two binders (sevelamer arm: mortality $15.0 / 100$ patient years, calcium arm: 16.1/100 patient years, HR $0.93(0.79-1.1) \mathrm{P}=0.4)$.

\section{References}

1 Dhingra R, Sullivan L, Fox CS, et al. Relations of serum phosphorus and calcium levels to the incidence of cardiovascular disease in the community. Arch Intern Med 2007; 167: 879-884

2 Block G, Klassen P, Lazarus J, et al. Mineral metabolism, mortality, and morbidity in maintenance hemodialysis. J Am Soc Nephrol 2004;15:2208-18

3 Covic A, Kothawala P, Bernal M, et al. Systematic review of the evidence underlying the association between mineral metabolism disturbances and risk of all-cause mortality, cardiovascular mortality and cardiovascular events in chronic kidney disease. Nephrol Dial Transplant 2009;24: 1506-1523.

4 Tentori F, Hunt WC, Rohrscheib M, et al. Which targets in clinical practice guidelines are associated with improved survival in a large dialysis organisation? J Am Soc Nephrol 2007;18:2377-2384

5 Suki W, Zabaneh R, Cangiano J, et al. Effects of sevelamer and calciumbased phosphate binders on mortality in hemodialysis patients. Kidney Int 2007;72:1130-1137 


\section{CKD-Mineral and Bone Disorders (Guidelines CKD-MBD 4.1-4.2)}

\section{Guideline 4.1 CKD-MBD: Serum PTH in patients with CKD $3 b-5$ (not on dialysis)}

We suggest that treatment is considered in patients with CKD stages $3 b-5$ not on dialysis therapy in whom serum PTH levels are progressively increasing and remain persistently higher than the upper reference limit for the assay, despite correction of modifiable factors. (2C)

\section{Guideline 4.2 CKD-MBD: Serum PTH in patients on dialysis (stage 5D)}

\section{Guideline 4.2.1 CKD-MBD: Target range of serum $P T H$ in patients on dialysis}

We suggest that the target range for parathyroid hormone measured using an intact PTH assay should be between 2 and 9 times the upper limit of normal for the assay used. $(2 \mathrm{C})$

\section{Guideline 4.2.2 CKD-MBD: Trends in serum PTH in patients on dialysis}

We suggest that marked changes in PTH levels in either direction within this range should prompt an initiation or change in therapy to avoid progression to levels outside this range. (2C)

\section{Rationale (for 4.1-4.2)}

These recommendations are in concordance with KDIGO [1]. To date, no randomised controlled trial has examined whether treatment to achieve a specific PTH target will improve outcomes. In the pre-dialysis

\section{References}

1 KDIGO Clinical Practice Guideline for the Diagnosis, Evaluation, Prevention, and Treatment of Chronic Kidney Disease-Mineral and Bone Disorder. Kidney Int 2009;76(Suppl 113):S1-132

2 Block GA, Klassen PS, Lazarus JM, et al. Mineral metabolism, mortality, and morbidity in maintenance hemodialysis. J Am Soc Nephrol 2004; 15:2208-2218

3 Block GA, Hulbert-Shearon TE, Levin NW, et al. Association of serum phosphorus and calcium $\times$ phosphate product with mortality risk in chronic hemodialysis patients: a national study. Am J Kidney Dis 1998; 31:607-617

\section{Acknowledgements}

Dr Simon Steddon has no conflicts of interest to declare. setting, optimal PTH concentration is unknown and it is sensible to evaluate those with values outside the reference range for contributory factors, such as hyperphosphataemia, hypocalcaemia, and 'nutritional' vitamin D deficiency. Treatment of these factors may reduce $\mathrm{PTH}$ levels toward the reference range, or prevent further increases. Serum alkaline phosphatase may also provide useful information on bone turnover and response to therapy. If PTH levels increase progressively and remain higher than the reference range, treatment with active vitamin D is likely to be necessary.

In the dialysis population, the PTH target focuses on avoidance of risk at extremes of $\mathrm{PTH}$; i.e. at $<2 \times$ or $>9 \times$ the upper limit of the normal reference range. The 'multiplication' reference range accommodates the significant variation in PTH levels between assays and laboratories. Intact PTH levels do not consistently predict bone histology, particularly if considered in isolation. The level at which PTH becomes significantly associated with increased all cause mortality varies among studies from $400-600 \mathrm{pg} / \mathrm{mL}$ [2-6]. The suggested PTH range corresponds to approximately $130-600 \mathrm{pg} / \mathrm{mL}$, depending on the assay used. There will not be uniform agreement around this target and we note concerns being less stringent may risk refractory hyperparathyroidism, but it seems appropriate to harmonise guidelines on the basis of available evidence. The percentage of CKD stage $5 \mathrm{D}$ patients undergoing parathyroidectomy is proposed as an audit standard.

It is clear that further studies to stratify risk are essential, and that these will need to focus on combinations of various biochemical deflections, rather than any particular parameter in isolation.

4 Kalantar-Zadeh K, Kuwae N, Regidor DL, et al. Survival predictability of time-varying indicators of bone disease in maintenance hemodialysis patients. Kidney Int 2006;70:771-780

5 Kimata N, Albert JM, Akiba T, et al. Association of mineral metabolism factors with all-cause and cardiovascular mortality in hemodialysis patients: the Japan dialysis outcomes and practice patterns study. Hemodial Int 2007;11:340-348

6 Young EW, Albert JM, Satayathum S, et al. Predictors and consequences of altered mineral metabolism: the Dialysis Outcomes and Practice Patterns Study. Kidney Int 2005;67:1179-1187

Dr Ed Sharples has received sponsorship from Amgen to attend educational meetings. 